Elsevier required licence: (C) 2018

This manuscript version is made available under the CC-BY-NC-ND 4.0 license http://creativecommons.org/licenses/by-nc-nd/4.0/

The definitive publisher version is available online at

10.1109/SeGAH.2018.8401317 


\title{
Assessing the Validity of In-Game Stepping Performance Data from a Kinect-based Fall Prevention Exergame
}

\author{
Jaime A. Garcia \\ UTS Games Studio \\ Faculty of Engineering and Information Technology \\ University of Technology Sydney \\ Sydney, Australia \\ Jaime.Garcia@uts.edu.au
}

\begin{abstract}
One of the main limitations of commercial games is the inability to determine improvements in the mental and physical health of the players. Although high game scores might provide an indication of higher cognitive and physical abilities, these are not sufficient to reliably determine the improvement in health outcomes. The work presented in this paper hence focuses on determining whether the collection of clinical measures during gameplay could potentially be used as a reliable indicator of improvement. For this study, the author uses the StepKinnection game for fall prevention, a Kinect-based game which builds on a hybrid version of the Choice Stepping Reaction Time (CSRT) task, a validated test that has been shown to prospectively predict older fallers. A group of 10 independent-living older adults was recruited and asked played with the game for a 12 weeks period of time. Assessments were conducted at baseline and every four weeks. Stepping performance data collected through gameplay was compared to the validated CSRT test. Statistical analysis proved that the stepping performance data collected by the game correlated and agreed with the validated measures of the CSRT test, suggesting that this could be used as a reliable indicator of health improvements.
\end{abstract}

Keywords-Fall prevention, elderly, older people, games for health, clinical data collection, Kinect.

\section{INTRODUCTION}

One in three people over 65 years old experience a fall every year [1]. Falls can have severe consequences ranging from bruises, fractures, traumatic brain injuries and even death [2]. The risk of falling can be reduced significantly by training specific cognitive and motor functions associated with balance, coordination, the ability to take quick reactive steps among others [3]. For example, exercise programs that include a component can potentially reduce the risk of falling by $40 \%$ [4]. The problem such programs is that the levels of adherence and engagement are very low. This is mostly due to the repetitive nature of the exercises that are often unattractive to the aged cohort

The use of interactive games has seemed to tackle this issue as such games offer playful contents that require full body motion to be able to play [5]. This can be considered a form of exercise.
In the past decade, balance games such as the Nintendo Wii Sports [6], the Nintendo Wii Fit [7] and stepping games such as Stepmania [8] and Dance Dance Revolution [9] have been used in several large clinical trials to determine whether commercial games can be used as a means to reduce the risk of falling. These studies have reported positive results in the physical health as well as high levels of compliance. Overall participants found the games very relevant, challenging and also enjoyable. Most importantly, subjects reported significant improvements in their walking speed, dynamic balance, mechanical lower limb muscle function, to name a few.

One of the limitations of this approach is the ability to reliably determine health improvements based on gameplay. A common generalization is that obtaining high game scores is directly related to the improvement of cognitive and motor functions. However, this can lead to misleading conclusions as the scoring system in these games are not necessarily in alignment with the therapeutical goals of a fall prevention intervention.

In order to reliably assess these changes, it is necessary to make use of validated clinical tools such as the Timed Up and Go test [10], 5 Sit to Stand test [11], or the Choice Step Reaction Time task [12], among others. Powerful tests that in the past have shown to reliably assess the risk of falling in the senior community as well as discriminate between fallers and non-fallers.

Traditionally, these tests are administered by therapists and researchers prior and post-intervention, which is often tedious, time-consuming task and in some cases inaccurate.

In a prior study, the authors presented the StepKinnection system [13]. A custom-made Kinect game that aims to address this issue by delivering stepping exercises and simultaneously collecting stepping performance data during gameplay. This allows for an automated fall risk assessment that can assist therapist and researchers to determine whether an intervention is being successful in a more accurate and time-effective manner. The work presented in the paper hence focuses on assessing the validity and accuracy of the clinical data collected by this game and whether it can be used as a reliable measure of improvement. This is achieved through a 
longitudinal study conducted over 10 weeks in which stepping performance data collected during gameplay is compared with a well-established fall prevention test.

The next section presents related work on the use of commercial games for falls prevention in large clinical trials. The StepKinnection game is then described in detail in Section 3 , along with a discussion of the design choices made to encourage key physical movements. Section 4 and 5 present the experimental methodology and the data collection, while Section 6 and 7 present the results of the study in relation to the validity and reliability of the stepping performance data collected during gameplay. Finally, a discussion of the implications of this work and potential future work are presented in Sections 8 and 9.

\section{Using COMmercial Games For FAll Prevention}

Physical activity and structured exercise have been shown to reduce the risk of developing several chronic diseases [14]. Programs targeting the lower limbs have been demonstrated to improve several dimensions associated with falling such as gait speed and balance coordination [15]. These exercises are more effective when these challenge balance through the inclusion of tasks that involve moving the centre of mass, reducing the base of support and reducing the use of upper limbs to maintain a balanced position [16].

For example, in the work done by Bateni et al [7], a 4-week Randomised Control Trial on 17 participants aged 53-91 using the Nintendo Wii Fit balance games (ski slalom, ski jump, table tilt) showed significant improvements in the static and dynamic balances. To play these games, subjects are required to shift weight in all directions in order to avoid obstacles presented on the screen. At the end of the study, all subjects reported significant improvements in the Berg Balance Scale Test (BBS) [17] and there were trends for improvement in the Bubble test for postural control.

Similarly, in the work done by Lamoth et al [6], 9 participants played with a balance control platform which requires them to use body tilt to guide a ball through a maze. This was a simple group quasiexperimental setup where participants had exposure to this system three times a week, for 20 mins over 6 weeks. Balance performance was determined through the use of a clinical measuring device and the Dot test for Postural Control. Pre and post comparisons showed significant improvements across the subjects. Performance on the dot task improved within the first two weeks. At the end of the intervention, task performance and balances were higher than at baseline. Subjects also reported high levels of satisfaction and enjoyment.

Step training has also shown improvements in dynamic balance and balance confidence, both associated with falling [18]. Stepping requires the integration of several dimensions such as sensory, central and neuromuscular systems. By taking a short proactive or reactive step an individual can increase their base of support regaining balance. Training this ability is important because it could be the last resort to prevent a fall from happening.

In the work done by Pichierri et al [8], 31 participants (mean age 86) played with a modified version of the game stepping game StepMania. In this game, arrows pointing in all directions scroll from the bottom to the top of the screen. Players need to step on the corresponding panels when such arrows reach certain level on the screen. The game was modified to allow for the control of the speed in which these arrows are presented and to allow for the collection of foot placement accuracy. Subjects played twice a week for 40 mins over a period of time of 12 weeks. Pre and post comparisons showed that the group that played with the game reported faster stepping times. Also, the fast walking performance under dual task conditions was significantly higher in the intervention group.

So far, the use of interactive games available in the market has shown positive results in engaging the elderly in physical exercise and rehabilitation. There are however some limitations as such games have not been specifically designed to provide therapeutic support for the senior community. Factors such as poor user interfaces, tasks that are not in alignment with recognized fall prevention exercises, and the inability to reliably determine changes in the mental and physical health throughout an intervention, make it difficult to use commercial games in unsupervised home-based settings on older people.

In order to address these issues, a bespoke Kinect-based exergame was developed to provide a user-friendly stepping game for older people to play at home [13]. This interactive step training system combines: (1) Appropriate age-related features to suit the needs and preferences of the elderly. (2) Meaningful tasks and exercise routines that are aligned with this health problem. (3) The collection stepping performance data to validate the effectiveness of the intervention. The combination of these three aspects makes the game potentially useful as a training program as it (1) is appealing to and easy to use by an elderly audience, (2) trains specific physical and cognitive functions associated with falls, and (3) allows for a continuous assessment of health outcomes from gameplay data.

In a prior study the authors assessed the feasibility and safety of using the game to deliver unsupervised step training exercises in older people's homes and as well as the efficacy of this intervention in improving stepping ability as well as physical and neuropsychological factors associated with falls in older people [19]. More recently, the user experience and engagement, usability and acceptance of the game was evaluated [20].

In this paper, we focus on assessing the accuracy of the clinical data collection during gameplay and whether this can be used as a reliable indicator of effectiveness in a fall prevention intervention.

\section{The StepKinnection GAme}

In the StepKinnection game, the player is an explorer who travels around the globe visiting a wide range of countries, 
hunting for treasures and seeking different adventures [13]. The further they travel, the more the difficulty increases. Gameplay starts with a series of basic levels where players will have the chance to become familiar with the game and coordinate their movements accordingly. Once they have finished these levels, players can move up to more challenging ones related to speed, precision, and cognitive complexity. In order to play the game, the player needs to stand in front of the TV facing the Kinect, as shown in Figure 1. Shortly after, the main menu will be presented, where players can select from a list of counties to visit. The player can wave either hand to move the cursor to navigate the menu. Once the level is selected the user moves to the main stepping tasks.

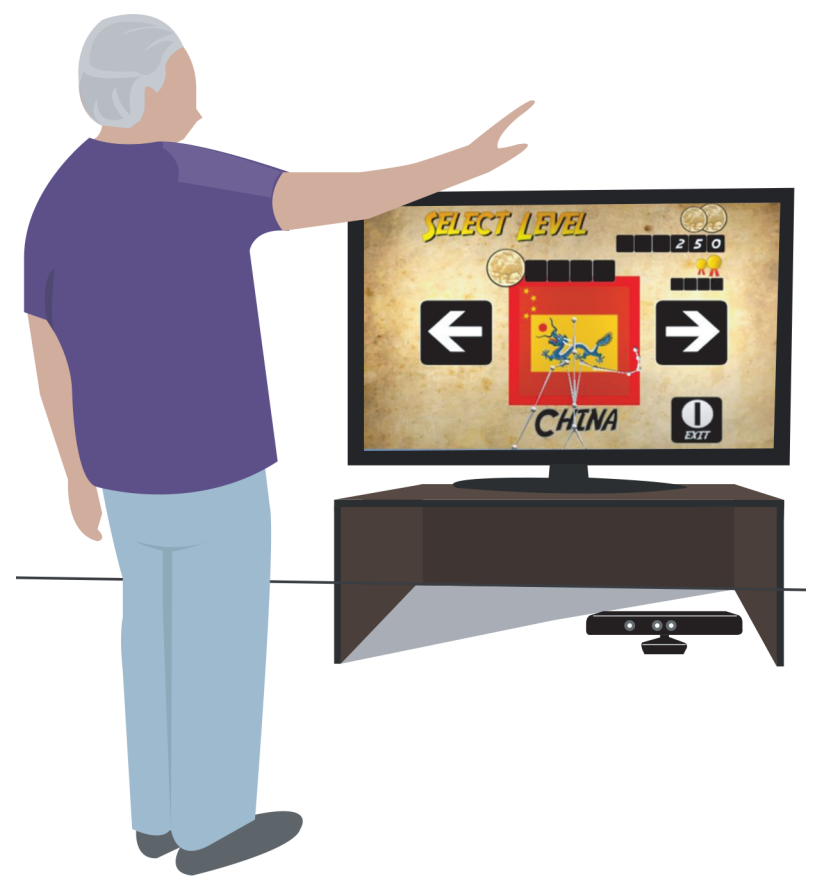

Figure 1. Navigating through the main menu.

\section{A. Stepping Tasks for Developing Motor Skills}

There are three main stepping tasks within the game, each designed to target a specific scenario and train the corresponding motor skills. These three activities include normal stepping, motor inhibition, and quick stepping.

For the normal stepping scenario, throughout the game fruits will appear in different areas of the screen every now and then, and the player is expected to collect them (See Figure 2). In order to achieve this, the player needs to reach the fruits by stepping on them, with steps being registered by the Kinect sensor. As the user moves through the levels, the time between the appearance of the fruits and the size of the fruits decrease. This is to encourage players to perform quicker and more accurate steps as they advance to higher levels, representing an increase in both physical capability and game-specific skills. This mechanism reinforces the ability to take proactive steps that could help an individual to regain balance and avoid falls. Also, as the stepping area decreases, the user needs to be more coordinated to be able to step on the fruits.

In addition to this standard stepping routine, a motor inhibition task was incorporated with the purpose of adding varying difficulty to the game by slightly increasing the cognitive demand. Training this ability is therefore ideal for situations where avoiding an obstacle can prevent a fall from occurring [21]. In the mid-levels, a ladybug might randomly appear on the screen. Stepping on the ladybug will take two penalty points off the current score, reducing the chances of winning. However, if the player remains in position, one point will be awarded.

Finally, in the higher levels, dollar coins randomly appear on the screen for a split-second. These coins are bonus points that can help players to move faster in the game, with the purpose of encouraging them to step faster. For each dollar coin that they collect, two bonus points will be added to their current score to reward the player, which motivates them to take quicker steps and improve their reaction time.

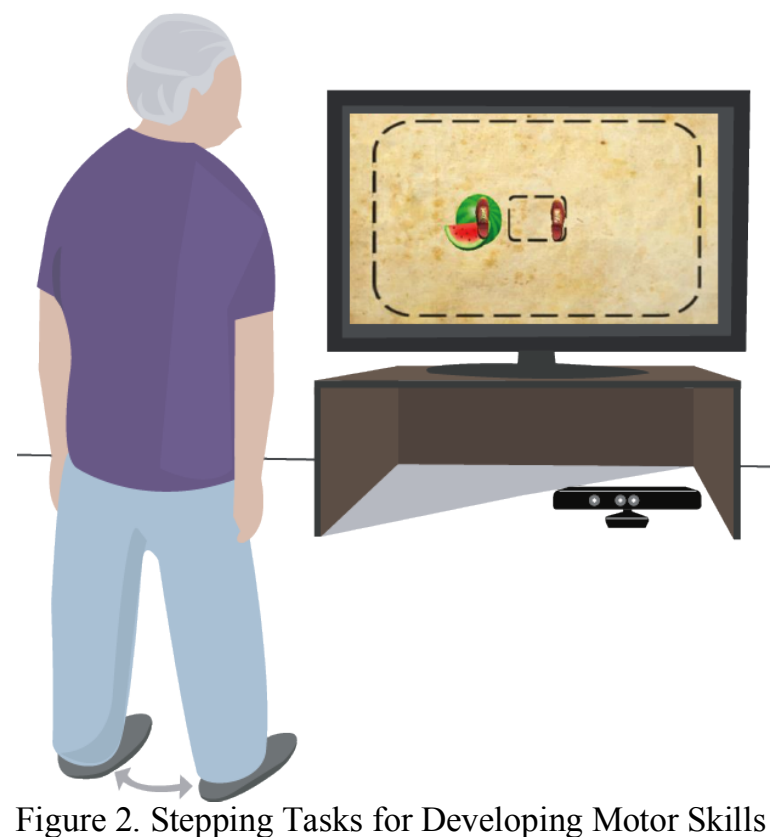

For all the above stepping tasks in the game, the accuracy of the responses is automatically processed by the hybrid clinical test for fall risk assessment that is embedded in the game. This is described in more detail in the next section.

The following section sets out the methodology used for this evaluation.

\section{Methodology}

For this study, 10 participants aged 65 years and over were recruited ( 8 females), with ten residents from two retirement villages in Sydney and two independent-living older adults.

Eligibility was determined by the following inclusion criteria: lives independently in the community, aged 65 years 
and over, able to walk independently without assistance, fluent in the English language, has a flat screen TV (LCD, LED or Plasma) with HDMI Port, internet connection (ideal), able to watch TV with their glasses from $3 \mathrm{~m}$ away, has enough room for system use $(1.2 \mathrm{~m}-2.5 \mathrm{~m})$, no major cognitive impairments.

Written informed consent was obtained from all participants prior to their participation in this study. Also, this research was approved by the University of New South Wales Human Research Ethics Committee (UNSW HREC HC12316) and ratified by the University of Technology Sydney Human Research Ethics Committee (UTS HREC 2012-279R).

Participants were given instruction on how to play the game and how to troubleshoot basic technical difficulties, mostly related to the use of the Kinect. Also, they were given a User Guide booklet and the contact details of the researchers for technical assistance. They were also encouraged to play as much as they wish. A minimum of three sessions of 20 minutes per week was the recommended dose. Participants exercised in their own homes with no supervision.

\section{DATA COLLECTION AND ANALYSIS}

In order to determine whether this can be used as a reliable indicator of health improvements, stepping performance data collected by the game was compared with stepping performance measured by a validated test for fall risk. For this study, a longitudinal study approach was selected. This involves repeated observations of the same variables over a long period of time, in this case, 12 weeks.

Data points were collected at baseline and every four weeks. Other clinical tests were also conducted in order to assess changes in the physical health and are reported in more detail in [19]. Also, bespoke and validated usability questionnaires were used to assess the user engagement and usability. These are reported in more detail in [20].

The following outcome variables were collected and analyzed:

\section{A. MAT measure of the Choice Stepping Reaction Time (CSRT) Test}

The CSRT test is a composite measure of sensorimotor functions, such as balance and strength, and cognitive functions such as attention and central processing speed. The test is able to combine several dimensions of fall risk based on these composite metrics. In the current version, the test is achieved through the use of a custom-made dance mat (or MAT) paired with an LCD monitor [22] (see Figure 3). The person stands on the two central step panels of the mat while facing the LCD monitor. Visual stimuli are presented on the LCD monitor and the user is expected to step accordingly on respective positions on the MAT. For this test, one of six surrounding panels illuminates on the LCD display and the person is required to step on this panel as quickly as possible and then return to the centre on the MAT. The sequence is presented randomly as well as the time between trials so that the user is unable to anticipate the time and location of the next stimulus. The mean reaction time of 36 trials is then measured and analysed.

\section{B. Kinect measure of the Choice Stepping Reaction Time (CSRT) Test}

For the purpose of this study, a Kinect version of the CSRT test was also used [23]. In the Kinect-driven system, the person stands in front of a computer screen or TV connected to a Kinect PC. The representation of the player in the system is a pair of shoes mirroring the person's feet movements. Six symmetrically distributed square-shaped virtual panels are drawn on the screen representing the step panels surrounding the person. The mechanics of the test are the same as the MAT version of the CSRT with the exception that the person steps in space.

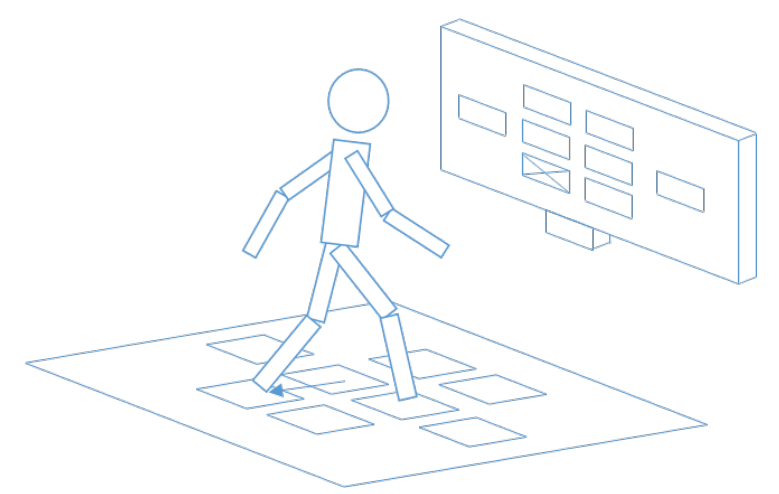

Figure 3. MAT version of the Choice Stepping Reaction Time test

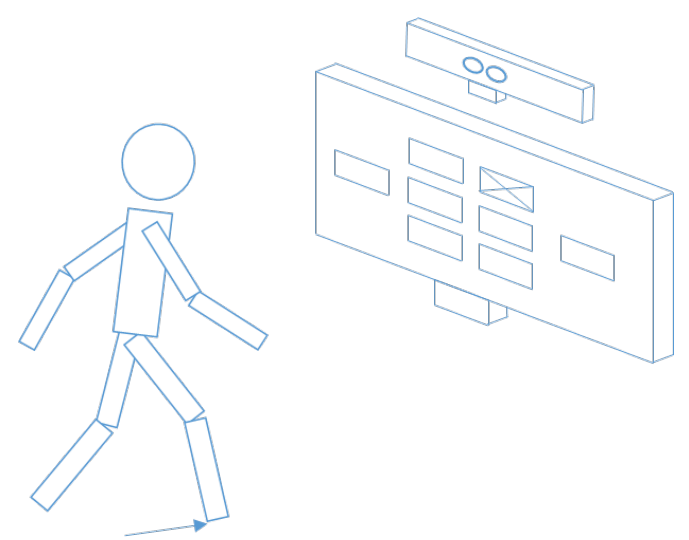

Figure 4. Kinect version of the Choice Stepping Reaction Time Task

\section{In-Game Measure of the CSRT}

During gameplay, the system automatically validates stepping responses and computes the average time taken by participants when reacting to stimuli are presented on the screen. To achieve this, the mechanics of the original CSRT test were incorporated into the game. User's actions such as a 'step' or a 'foot liftoff' are recognized by Kinect's depth sensor. These actions are subsequently used for the calculation 
of the following time-based variables which are essential in the calculation of the CSRT test: (1) Decision Time (DT): time elapsed between the instance where the fruit appears on the screen and the player lifts his/her foot off the centre, (2) Movement Time (MT): time it takes for the user to step on a fruit once leg movement is initiated; (3) Response time (RT): Decision Time (DT) + Movement Time (MT). Since the collection of this data is performed on an ongoing basis, the average reaction times were used for the comparison. This is explained in more detail in [23].

In order to determine the validity of the stepping performance data collected by the StepKinnection game, this was compared with the CSRT measurements collected throughout the assessments. Friedman's 2-way analysis of variance (ANOVA) by ranks was used to determine differences between assessments, with tests to determine which between-group differences were significant. Consistency and association were assessed through the Pearson Correlation Coefficient (Derrick et al., 1994) and the Intraclass Correlation Coefficient model 3,1 (ICC3,1). Finally, the level of significance was set at 5\%. Analyses were conducted using SPSS for Windows (Version 20).

\section{RESULTS}

The MAT measure of the CSRT test showed significant improvements. The Friedman ANOVAs model indicated that there was a significant difference between the four assessments $\left(\mathrm{X}^{2}=16.879\right.$, df $\left.=3, \mathrm{p}=0.001, \mathrm{n}=10\right)$. $\mathrm{A}$ Wilcoxon signed-rank test showed noticeable improvement between the baseline assessment and the first month of intervention $(Z=-2.803, p=0.005)$. Also, post-hoc assessments showed trends for improved stepping time performance between baseline and assessment $1(\mathrm{Z}=-1.886, \mathrm{p}$ $=0.059)$, and baseline and assessment $2(\mathrm{Z}=-2.803, \mathrm{p}=$ $0.005)$ and baseline and assessment $3(Z=-2.701, p=0.007)$.

Similarly, the Kinect version of the CSRT test reported similar results across the participants. Despite the technological differences between these two platforms, the Friedman ANOVAs model indicated that there was a significant difference between the four assessments $\left(X^{2}=12.24\right.$, df $=3, p$ $=0.007, \mathrm{n}=10)$. A Wilcoxon signed-rank test showed noticeable improvement between the baseline assessment and the first month of intervention $(Z=-2.293, p=0.022)$. Also, post-hoc assessments showed trends for improved stepping time performance between baseline and assessment $1(\mathrm{Z}=$ 1.886, $\mathrm{p}=0.059)$, and baseline and assessment $2(\mathrm{Z}=-1.784$, $\mathrm{p}=0.074)$.

Regarding the validity of the stepping performance data collected during gameplay, Pearson Correlation Coefficient showed a fair association between the reaction time values collected by the game and the monthly performed Kinectbased CSRT test (Pearson $r=0.624, p=0.000$ ). Likewise, a weak positive correlation was found between the reaction time values collected by the game and the monthly performed MAT-based CSRT test (Pearson $\mathrm{r}=0.329, \mathrm{p}=0.000$ ).
Additionally, the ICC model 3,1 showed consistency and agreement between these values across the participants throughout the intervention (ICC Single Measures $=0.597$, Average Measures $=0.747, \mathrm{p}=0.000)$.

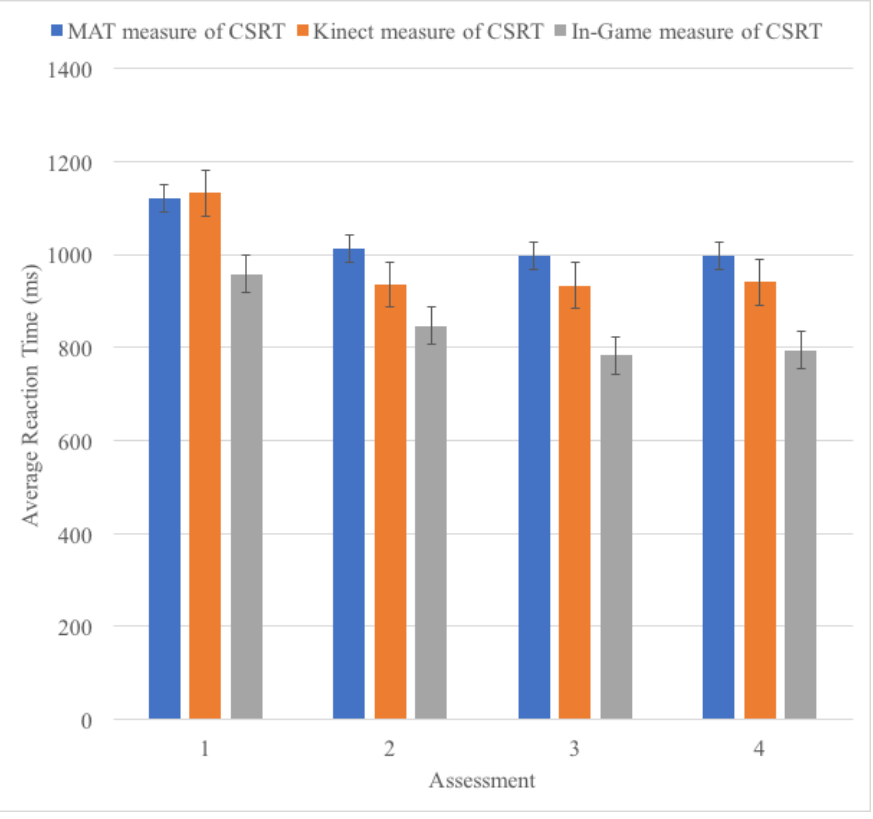

Figure 5: Results: Comparison between the MAT measure of the CSRT test, the Kinect measure of the CSRT and the InGame measure of the CSRT test.

\section{FINDINGS}

Significant changes in the reaction times registered by both the Kinect and MAT version of the CSRT test were found, indicating improvements in the participants' central processing speed and movement velocity.

More importantly, statistical analysis demonstrated that the stepping performance data collected by the game correlates and agrees with the traditional measure of the CSRT, suggesting that this could be used as a reliable indicator for health improvements (see Figure 5). Consequently, this measure was assessed to determine whether there were statistically significant changes among the participants. Results showed a significant improvement of $17 \%$ across the 10 participants with a high level of significance $(p=0.000)$, which is the same percentage of improvement reported by the Kinect CSRT test. Also, this value was found to be in agreement with a prior long-term study where the use of a stepping mat device showed a similar rate of improvement in the stepping test [24]. The results suggest that this measure is a reliable indicator to determine improvements in central processing speed and movement velocity. 
More importantly, the mean improvement of $164 \mathrm{~ms}$ determined by the CSRT measure obtained during gameplay was found to be clinically meaningful as this is very similar the $150 \mathrm{~ms}$ difference in CSRT times that discriminates between multiple fallers and multiple non-fallers [22]. All these suggest that the ability to collect these measures simultaneously makes this system potentially useful in a clinical setting as it can evaluate several dimensions involved in the assessment of fall risk in older people in a nonobtrusive, time-effective and fully automated manner.

\section{DISCUSSION}

One of the main limitations of commercial games is the inability to determine improvements in the mental and physical health of the players. Although high game scores might provide an indication of higher cognitive and physical abilities, these are not sufficient to reliably determine the improvement in health outcomes. For that reason, it is crucial to incorporate mechanisms that can measure clinically validated parameters during gameplay. This not only allows for the validation of the correct exercise movements but also allows for the assessment of physical and mental functions in order to determine progress and health improvements on an ongoing basis. In this study, the stepping performance data collected through the StepKinnection game was compared to the validated version of the CSRT test for fall risk assessment. In addition to this, the data presented show that the In-Game measure of the CSRT test was overall shorter than the traditional tests, suggesting that this test might be slightly less cognitively demanding. It is likely that the immersive nature of this system and the appropriate provision of real-time feedback remove the extra processing time required to operate with the MAT (where stimuli are presented on the screen and the execution is expected to happen on the dance mat). Alternatively, participants may have felt more confident to step faster in space due to the absence of wearable sensors or a physical apparatus.

Based on the obtained results, the 12-week intervention with the Kinect-based stepping game at home significantly improved several cognitive and physical functions that have been shown to be associated with high risk of falling in older people. The 10 participants also indicated that they enjoyed the stepping training and found it useful to improve their health. One of the main limitations of this study is the small sample size that made it difficult to generalize our findings. Also, due to complications during the recruitment stages, it was not possible to set up and monitor a control group for comparisons pre and post-intervention. Despite this, the findings are still considered relevant and clinically meaningful as the majority of the outcome measures presented similar levels of improvements to several studies found in the academic literature.

\section{CONClusions ANd Future WORK}

This paper presents the results of a 12-week intervention to evaluate the accuracy and reliability of stepping performance data collected during gameplay. Since the main interest of this study is to determine whether such data can be used as a reliable indicator of health improvement and progression, a convenient sample of 10 older adults was recruited and asked to play with the StepKinnection game for 12 weeks. Stepping performance data was collected by the game was compared with stepping performance data obtained through the use of validated clinical tests. Statistical analysis showed that the collection of these clinical parameters during gameplay was found to be a reliable indicator of health improvement. These parameters not only correlated well with a validated version of the CSRT test but also showed significant improvements among the participants as a result of playing with the stepping game. These results overcome the main limitation in commercial games that are only designed for the purpose of entertaining a younger audience. Incorporating mechanisms that can obtain clinically meaningful parameters during gameplay allows for assessment of physical and mental functions on an ongoing basis and the determination of progress in an unobtrusive manner. More importantly, the 12week intervention study also showed that a game mindfully designed for the elderly, with specific health alignments and an embedded health assessment tool, can significantly reduce the risk of falling in the elderly. The continuation of this work includes studies with larger sample sizes in order to confirm these findings and determine if secondary outcome measures that did not show significant changes among the participants can be improved.

\section{REFERENCES}

1. Masud, T. and R.O. Morris, Epidemiology of falls. Age and ageing, 2001.30(suppl_4): p. 3-7.

2. Aschkenasy, M.T. and T.C. Rothenhaus, Trauma and falls in the elderly. Emergency Medicine Clinics, 2006. 24(2): p. 413-432.

3. Maki, B.E. and W.E. McIlroy, Control of rapid limb movements for balance recovery: age-related changes and implications for fall prevention. Age and ageing, 2006. 35(suppl_2): p. ii12-ii18.

4. Barnett, A., et al., Community-based group exercise improves balance and reduces falls in at-risk older people: a randomised controlled trial. Age and ageing, 2003. 32(4): p. 407-414.

5. Pietrzak, E., C. Cotea, and S. Pullman, Using commercial video games for falls prevention in older adults: The way for the future? Journal of geriatric physical therapy, 2014. 37(4): p. 166-177.

6. Lamoth, C.J., S.R. Caljouw, and K. Postema, Active video gaming to improve balance in the elderly. 2011.

7. Bateni, H., Changes in balance in older adults based on use of physical therapy vs the Wii Fit gaming system: a preliminary study. Physiotherapy, 2012. 98(3): p. 211216.

8. Pichierri, G., K. Murer, and E.D. de Bruin, A cognitivemotor intervention using a dance video game to enhance foot placement accuracy and gait under dual task conditions in older adults: a randomized controlled trial. BMC geriatrics, 2012. 12(1): p. 74.

9. Smith, S.T., et al., A novel Dance Dance Revolution (DDR) system for in-home training of stepping ability: 
basic parameters of system use by older adults. British journal of sports medicine, 2011. 45(5): p. 441-445.

10. Shumway-Cook, A., S. Brauer, and M. Woollacott, Predicting the probability for falls in community-dwelling older adults using the Timed Up \& Go Test. Physical therapy, 2000. 80(9): p. 896-903.

11. Lord, S.R., et al., Sit-to-stand performance depends on sensation, speed, balance, and psychological status in addition to strength in older people. The Journals of Gerontology Series A: Biological Sciences and Medical Sciences, 2002. 57(8): p. M539-M543.

12. Lord, S.R. and R.C. Fitzpatrick, Choice stepping reaction time: a composite measure of falls risk in older people. The Journals of Gerontology Series A: Biological Sciences and Medical Sciences, 2001. 56(10): p. M627M632.

13. Garcia, J.A. and K.F. Navarro, StepKinnection: a fall prevention game mindfully designed for the elderly. Studies in health technology and informatics, 2015. 214: p. 43-49.

14. Chodzko-Zajko, W.J., et al., Exercise and physical activity for older adults. Medicine \& science in sports \& exercise, 2009. 41(7): p. 1510-1530.

15. Howe, T.E., et al., Exercise for improving balance in older people. The Cochrane Library, 2011.

16. Sherrington, C., et al., Effective exercise for the prevention of falls: a systematic review and meta-analysis. Journal of the American Geriatrics Society, 2008. 56(12): p. 2234-2243.

17. Muir, S.W., et al., Use of the Berg Balance Scale for predicting multiple falls in community-dwelling elderly people: a prospective study. Physical therapy, 2008. 88(4): p. 449-459.

18. Studenski, S., et al., Interactive video dance games for healthy older adults. The journal of nutrition, health \& aging, 2010. 14(10): p. 850-852.

19. Garcia, J.A., et al., A bespoke Kinect stepping exergame for improving physical and cognitive function in older people: A pilot study. Games for health journal, 2016. 5(6): p. 382-388.

20. Garcia, J., W. Raffe, and K. Felix Navarro. Assessing User Engagement with a Fall Prevention Game as an Unsupervised Exercise Program for Older People. in Proceedings of Australian Computer Science Week (ACSW 2018). 2018.

21. Potocanac, Z., et al., Response inhibition during avoidance of virtual obstacles while walking. Gait \& posture, 2014. 39(1): p. 641-644.

22. Schoene, D., et al., A novel video game-based device for measuring stepping performance and fall risk in older people. Archives of physical medicine and rehabilitation, 2011. 92(6): p. 947-953.

23. Garcia, J.A., et al. Assessing the Kinect's capabilities to perform a time-based clinical test for fall risk assessment in older people. in International Conference on Entertainment Computing. 2014. Springer.

24. Schoene, D., et al., A randomized controlled pilot study of home-based step training in older people using videogame technology. PloS one, 2013. 8(3): p. e57734. 